\title{
Efficient Intracavity Frequency Doubling of an Yb-doped Fiber Laser Using an Internal Resonant Enhancement Cavity
}

\author{
R. Cieslak, J. K. Sahu, W. A. Clarkson \\ Optoelectronics Research Centre, University of Southampton, Southampton, SO17 1BJ, UK \\ Authore-mail address: rxc@orc.soton.ac.uk
}

\begin{abstract}
We describe a simple approach for efficient generation of visible light in high-power continuous-wave fiber lasers via second harmonic generation in an internal resonant cavity. Preliminary results for a cladding-pumped $\mathrm{Yb}$ fiber laser are presented. (C)2010 Optical Society of America OCIS codes: (140.3510) Lasers, fiber; (140.3515) Lasers, frequency doubled
\end{abstract}

\section{Introduction}

High power laser sources emitting in the visible spectral region have a diverse range of applications in areas such as laser processing of materials, projection displays, medicine and sensing. For the continuous-wave (CW) operating regime the most popular approach is via intracavity second harmonic generation in a diode-pumped 'bulk' solidstate laser. This approach exploits the relatively low resonator losses and hence high intracavity powers that can be achieved in these lasers to achieve high second harmonic conversion efficiency [1]. Indeed, there are now a number of impressive commercial laser products based on intracavity second harmonic generation in diode-pumped $\mathrm{Nd}: \mathrm{YVO}_{4}$ lasers that can generate single-spatial-mode green output at $532 \mathrm{~nm}$ in the $10 \mathrm{~W}$ regime [2]. However, scaling to higher powers is rather more challenging due to the effects of heat generation in the laser medium which lead to degradation in beam quality and increased resonator loss. Fiber lasers from a geometry that is relatively immune to the effects of heat generation in the core and hence offer a route to much higher power levels in the nearinfrared wavelength regime via the use of cladding-pumped architectures [3], and hence offer the prospect of much higher power levels in the visible regime via nonlinear frequency conversion. Unfortunately, the technique of intracavity second harmonic generation is not well-suited to cladding-pumped fiber lasers since they have rather high resonator losses. One solution to this problem is to employ the technique of external resonant cavity second harmonic generation. This approach has been successfully applied to cw fiber sources [4], but suffers from the drawback of added complexity since a single-frequency fiber master-oscillator power-amplifier is required and the master-oscillator and/or resonant cavity lengths must be actively stabilised to ensure that the resonance condition is maintained at all times.

In this paper we describe a simple alternative approach for efficient second harmonic in high-power CW fiber lasers which offers the prospect of both very high conversion efficiency and very high power in the visible wavelength regime. The approach is based on intracavity second harmonic generation, but with an internal resonant enhancement cavity containing the nonlinear crystal to avoid the problems associated with the high fiber resonator loss. In this scheme, the fiber laser automatically lases on axial modes that are resonant in the enhancement cavity avoiding the need for active stabilisation of the cavity length and hence providing immunity from thermallyinduced and mechanically-induced variations in path length. A similar nonlinear frequency conversion scheme has previously been reported for low-power single-frequency semiconductor sources [5,6], but despite its obvious potential has not yet been applied to high power fiber lasers operating on many axial modes to the best of our knowledge. Here we present preliminary results for a cladding-pumped ytterbium (Yb)-doped fiber laser frequencydoubled to $530-550 \mathrm{~nm}$ (green) wavelength regime and consider the prospects for further improvement in performance.

\section{Experiment and results}

The Yb fiber laser configuration used in our experiments (shown in Fig. 1) comprised a relatively standard doubleclad $\mathrm{Yb}$-doped silica fiber with a single-mode core of diameter, $9.6 \mu \mathrm{m}$ and a pure silica inner-cladding of diameter, $150 \mu \mathrm{m}$. Feedback for lasing was provided by an external cavity containing a simple diffraction grating at one end of the fiber and, at the opposite end, by another external feedback arrangement containing the resonant enhancement cavity. The diffraction grating was used to select the fiber laser's operating wavelength and to narrow the emission bandwidth so that it was less than the phase-matching bandwidth for the nonlinear crystal. Pump light from a fiber-coupled diode-bar at $975 \mathrm{~nm}$ was launched into the fiber end adjacent to the diffraction grating with the aid of a dichroic mirror. A simple bow-tie resonator design was used for the enhancement cavity. The latter 
comprised two plane-plane mirrors with reflectivities of $97.5 \%$ and $98.5 \%$ at the fundamental wavelength and two concave mirrors (75 mm radius of curvature) with high reflectivity $(>99.8 \%)$ at the fundamental wavelength and high transmission at the second harmonic wavelength, and a $15 \mathrm{~mm}$ long Brewster-angled $\mathrm{LiB}_{3} \mathrm{O}_{5}$ (LBO) crystal placed in an oven and cut for type I non-critical phase-matching. The external feedback arrangement was terminated by a plane high reflectivity mirror. In this scheme the fundamental laser power is enhanced in the resonant cavity by virtue of its relatively low loss. Hence with optimum operating conditions and careful mode-matching of the $\mathrm{Yb}$ fiber output to the $\mathrm{TEM}_{\mathrm{oo}}$ mode of the internal resonant cavity, this arrangement can yield a high second harmonic conversion efficiency. The diffraction grating could be adjusted to tune the fundamental wavelength in the range $1060-1100 \mathrm{~nm}$ (with maximum power at $\sim 1085 \mathrm{~nm}$ ) yielding second harmonic output in the range $530-550 \mathrm{~nm}$ with oven temperature adjusted for phase-matching at the relevant wavelength (e.g. $\sim 122.5{ }^{\circ} \mathrm{C}$ for $1085 \mathrm{~nm}, \sim 150{ }^{\circ} \mathrm{C}$ for $1064 \mathrm{~nm})$.

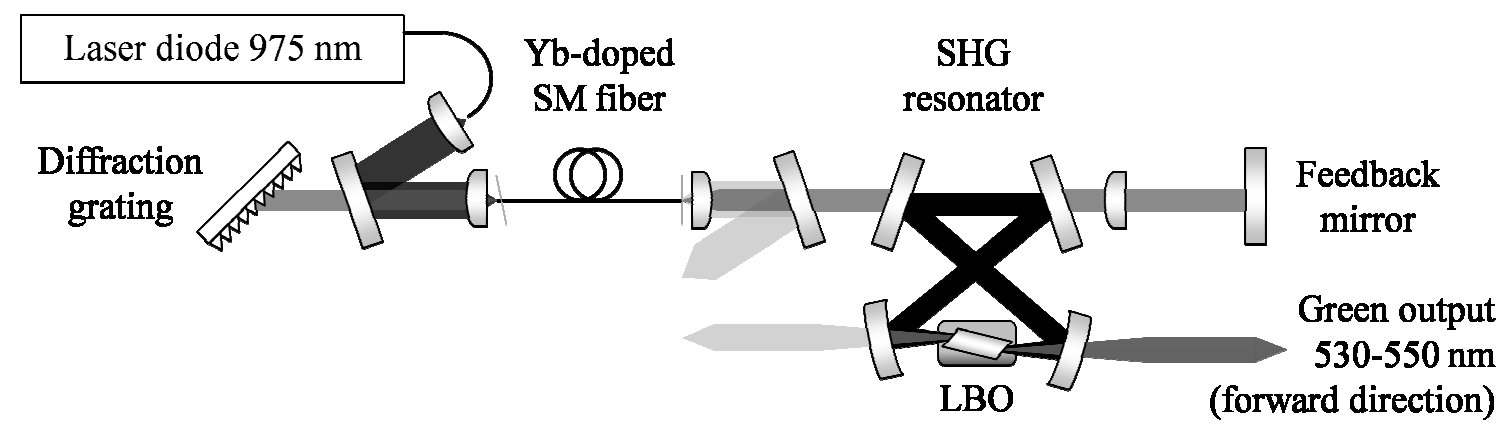

Fig. 1. Experimental set-up for efficient frequency doubling in a fiber laser

Using this arrangement we obtained $\sim 1.3 \mathrm{~W}$ (i.e. $\sim 1.6 \mathrm{~W}$ inside the LBO crystal) of CW output at $542.5 \mathrm{~nm}$ corresponding to $\sim 7.5 \mathrm{~W}$ of fundamental power incident on the internal resonant cavity. Due to imperfect modematching and impedance matching arising from limited availability of components only $\sim 4.9 \mathrm{~W}$ was actually coupled into the resonant cavity and hence $\sim 13 \%$ of the total infrared power $(\sim 13 \mathrm{~W})$ generated in the fiber was converted to green output in the forward direction. The second harmonic output backward direction was less than $50 \mathrm{~mW}$. The effective single-pass conversion efficiency was $\sim 2 \%$ at $1.3 \mathrm{~W}$ of green output corresponding to a resonant enhancement factor of $\sim 15$.

These preliminary results were achieved with a non-optimal set-up. The 'non-useful' cavity loss for the enhancement cavity at the fundamental wavelength was $\sim 3 \%$ and the spatial overlap factor between the resonant cavity mode and incident beam was $\sim 0.85$. Furthermore, $\sim 23 \%$ of spatially mode-matched fundamental power was rejected due to impedance mismatch. Further optimisation of the resonator design to reduce losses and improve mode-matching and impedance matching should yield a green power of $>6.5 \mathrm{~W}$ for the same pump power, corresponding to a second harmonic conversion efficiency with respect to total fundamental power of $>50 \%$. The green power can be further increased by employing a high-power pump source and improved Yb-doped double-clad fiber design.

\section{Conclusion}

In summary, we have demonstrated a simple approach for efficient frequency doubling in fiber lasers which should, with careful optimization, be applicable to very high power cladding-pumped fiber lasers allowing the generation of $\mathrm{CW}$ visible powers in the multi-hundred watt regime and beyond.

\section{References}

[1] K. I. Martin et al., "Stable, high-power, single-frequency generation at $532 \mathrm{~nm}$ from a diode-bar-pumped Nd:YAG ring laser with an intracavity LBO frequency doubler", Appl. Opt. 36 (18), 4149 (1997)

[2] http://www.newport.com/Millennia-Prime-532-nm-CW-DPSS-Lasers/501529/1033/catalog.aspx

[3] A. Liem et al., "1.3 kW Yb-doped fiber laser with excellent beam quality", in Proc. Conf. Lasers Electro-Opt. (Washington, D.C, 2004), pp. 1067-1068

[4] T. Sudmeyer et al., "Efficient 2nd and 4th harmonic generation of a single-frequency, continuous-wave fiber amplifier", Opt. Express, 16 (3), $1546-1551$ (2008)

[5] W. J. Kozlovsky et al., "Blue light generation by resonator-enhanced frequency doubling of an extended-cavity laser", Appl. Phys. Lett., 65, 525-527 (1994)

[6] P. G. Wigley et al., "High-power 467-nm passively locked signal-resonant sum-frequency laser", Opt. Lett., 20, 2496-2498 (1995) 